\title{
Factoring out Glue-code in Systems of IoT Devices
}

The PalCom Architecture Model

\author{
Boris Magnusson \\ Department of Computer Science \\ Lund University \\ Lund, Sweden \\ Boris.Magnusson@cs.lth.se
}

\author{
Björn A. Johnsson \\ Department of Computer Science \\ Lund University \\ Lund, Sweden \\ Bjorn_A.Johnsson@cs.lth.se
}

\author{
Görel Hedin \\ Department of Computer Science \\ Lund University \\ Lund, Sweden \\ Gorel.Hedin@cs.lth.se
}

\begin{abstract}
We present an architecture for distributed, heterogeneous systems where there is a clear separation of concerns for Computation, Coordination, Configuration. The computation part is often implemented in traditional programming code, and packaged as services. The other concerns might need to be addressed much later and by other people in separate organisations, or even by end users. In this paper we present the model used by the PalCom middleware where each of the concerns can be implemented separate, in separate representations, by separate people and yet combined and updated afterwards. The PalCom middleware is implemented in Java and in active use in research projects and in commercial products.
\end{abstract}

\section{CCS CONCEPTS}

- Computer systems organization $\rightarrow$ Peer-to-peer architectures; - Software and its engineering $\rightarrow$ Message oriented middleware; Programming by example; Visual languages.

\section{KEYWORDS}

IoT, Middleware, PalCom Architecture, interconnecting code, microservices, automatic update

\section{ACM Reference Format:}

Boris Magnusson, Björn A. Johnsson, and Görel Hedin. 2019. Factoring out Glue-code in Systems of IoT Devices: The PalCom Architecture Model. In Programming '19, April 1-4, 2019, Genova, Italy. ACM, New York, NY, USA, 4 pages. https://doi.org/10.1145/3328433.3328436

\section{INTRODUCTION}

The emerging use of computerised communicating devices has given rise to a number of new research fields, like ubiquitous computing, pervasive systems, and, more recently, internet-of-things. The devices, or "things", are often mobile, and communicate on a variety of network technologies and give rise to a new set of challenges for software development and maintenance [8]. Furthermore, we expect them to work together, and we, the users, would like to combine them quite freely. Mobile devices may move between network technologies, and sometimes be outside any network. Still,

Permission to make digital or hard copies of part or all of this work for personal or classroom use is granted without fee provided that copies are not made or distributed for profit or commercial advantage and that copies bear this notice and the full citation on the first page. Copyrights for third-party components of this work must be honored. For all other uses, contact the Owner/Author.

Programming '19, April 1-4, 2019, Genova, Italy

(C) 2019 Copyright is held by the owner/author(s).

ACM ISBN 978-1-4503-6257-3/19/04.

https://doi.org/10.1145/3328433.3328436 we expect communication and routing to be transparent, and that operation degrades gracefully when devices are temporarily out of reach.

Different devices provide different functionality, or services, that we would like to use and combine. There is a growing number of de-facto standard service protocols, used by vendors in different fields. They provide islands of compatibility, but offer no general interoperability. Despite this, we expect future applications to be built from component devices and services following different standards. We do not think that one future common standard is the answer (it will always be outdated), but rather that interoperability should be provided in a way that makes it easy to bridge over different protocols. For this we need meta standards that can be used to bridge standard protocols [3].

With forecasts of 26 billion connected devices by 2020 [4], the flat architectures of today, using client-server, point-to-point, and $\mathrm{TCP} / \mathrm{IP}$ connections, need to be complemented. There is a need for scalable architectures that support logical networks operating over heterogeneous physical networks, organised in substructures to hide details. We are long past the point where the internet could be re-booted in case of failures or upgrades, and IoT systems will even more call for architectures that can evolve, and support its parts to evolve.

The ways devices and configurations can be combined cannot always be foreseen in advance, but new creative solutions will emerge. This is a process that involves not only creators of devices and systems, but to a large degree the users themselves, at different levels of technical competence. The need for end-user composition will grow as the number of IoT systems grows, requiring a high abstraction level to reduce development effort. While some IoT systems may primarily automate functionality, and thus be invisible as in Weiser's original vision [9], there will be many IoT systems that are interactive. This will require the easy and high-level construction of user interfaces that combine functionality of several devices and services.

Building a new IoT system from the ground up is a labor intensive task with a large amount of low-level programming which can be largely reduced when using a suitable middleware. We have worked with pervasive systems for more than a decade, developing the PalCom middleware [7]. PalCom originates from the project Palpable Computing [6] and has been gradually evolved, with refined concepts to better support internet systems of things, and applied to different domains like healthcare, consumer electronics, and mobile robotics. 


\section{BACKGROUND}

The PalCom architecture has been influenced by the experience from the software maintenance community as it comes forward in Andrade et al [1] where the presentation of the three concerns computation, coordination and configuration are described. Coordination and configuration covers what is often described as "glue code" and the role is to interconnect and make existing components work together. In this paper we assume the mechanisms for transparent communication, discovery, routing and security that the lower layers of PalCom offer, to be available.

We motivate the proposed architecture by an example from real life. The problem of putting together a system of existing components is illustrated by the situation when connecting a computer to a projector. This problem can be solved with a cable and/or an adaptor. From the architecture perspective, we can view this as:

- Computer, Projector: offers computation, or services.

- Adaptor: offers coordination in case the services are not compatible (between VGA and HDMI e.g.).

- Cable: implements configuration by connecting the two services.

We note that: the two services do not know about each other, but the cable attached to both of them "knows" about them both With a suitable adaptor, we can combine services that are not made to work together. If we update one of the services we might need to use another, or an updated, adaptor to create a new version of the working system. The services come from different vendors and are created independently by different people, possibly separated in time. The adaptor is created with detailed knowledge of the two services it coordinates, often by another vendor, at a later time. It is thus essential that it is possible to provide adaptors (and cables) that can be placed between the services to meet changing conditions.

In general, this architecture works well compared to situations where the components need to be wired together, or are built to only work with specific other devices. The architecture offered by PalCom is trying to create a similar flexibility for putting together distributed software systems.

\section{THE PALCOM ARCHITECTURE MODEL}

Following the experience from [1] we have identified the following kinds of components in the PalCom architecture:

Device Computational equipment on a network that host and execute PalCom components such as services and assemblies.

Service Embeds computation, offers a communication interface specifying a set of commands (messages) it can send and/or understand. Each command can have parameters.

Assembly Embeds coordination, offers adaption of one or more services.

Configuration A set of components such as Services and Assemblies and embeds information about how the components are interconnected.

A Service can act as a gateway, facade, to an existing functionality external to the PalCom architecture, or it may be self-contained, i.e. include the functionality, or computation, it offers to the PalCom network.
An Assembly can be connected to one or more services, and in its turn offer services. It embeds the functionality, the glue, needed to make the connected services work together for a particular purpose. This includes acting on incoming messages from the services it is bound (connected) to, and produce zero or more messages in response to the connected services. An assembly includes default bindings to the original services used while the assembly is constructed as needed for the programming-by-example approach supported by PalCom tools. These original bindings are overridden by bindings to services on devices included in the configurations where the assembly is included. An assembly can take part in different configurations. Since assemblies can offer functionality as services they can be combined hierarchically.

A Configuration specifies which services and assemblies (and other configurations) it includes and how they are interconnected. It may in its turn have open bindings that need to be bound before or when it is deployed on a device. Several configurations can be deployed on the same device.

A system concept that can include configurations, (and possibly other systems), deployed on different devices is also needed, but remains to be specified. Currently one have to manually make sure that communicating devices use compatible versions of components, such as services and assemblies. Additionally, there are specific components that we want to mention, but do not describe further here; GUI-descriptions, Web-content components, etc. that also embed computation and honor the PalCom description protocol for services [3].

\section{A SMALL EXAMPLE - THE CONNECTED KETTLE}

In this small example we want to illustrate the roles of the components in the PalCom architectures by building a remotely controlled kettle from pre-existing components. Such a set-up could be useful for making sure you have hot water for tea without delay as you get home. We have an electric kettle, a remotely controlled power plug with USB adapter and associated software from TellStick, a thermometer with USB-plug and associated software from 1-Wire, and a Raspberry Pi Zero computer with the PalCom tool, TheThing, as shown in Figure 1.

On this TheThing we deploy two services, Boiler and Temp, that work as gateways to the TellStick and 1-Wire software systems respectively. We also deploy two self-contained services as shown in Figure 1: Comparator and Clock. These services are represented as yellow rectangles in the figure.

The Boiler service provides commands On and Off to control the power to the kettle. The Temp service can be polled for the current temperature in the kettle (commands getTemperature and Temperature). The service Clock can be set to generate periodic Tick-commands, in this case every 3 seconds. Comparator can be configured with two limits (high, low) and will then respond to a CheckValue command and its parameter temp, with a ValueOverRange (temp $>$ high), ValueUnderRange (temp $<$ low) or with no response in case temp falls between the to limits (high>temp>low).

All these services can be connected to and the functionality explored by the PalcomBrowser development tool. This tool embed a function that can interpret the interface descriptions the services 


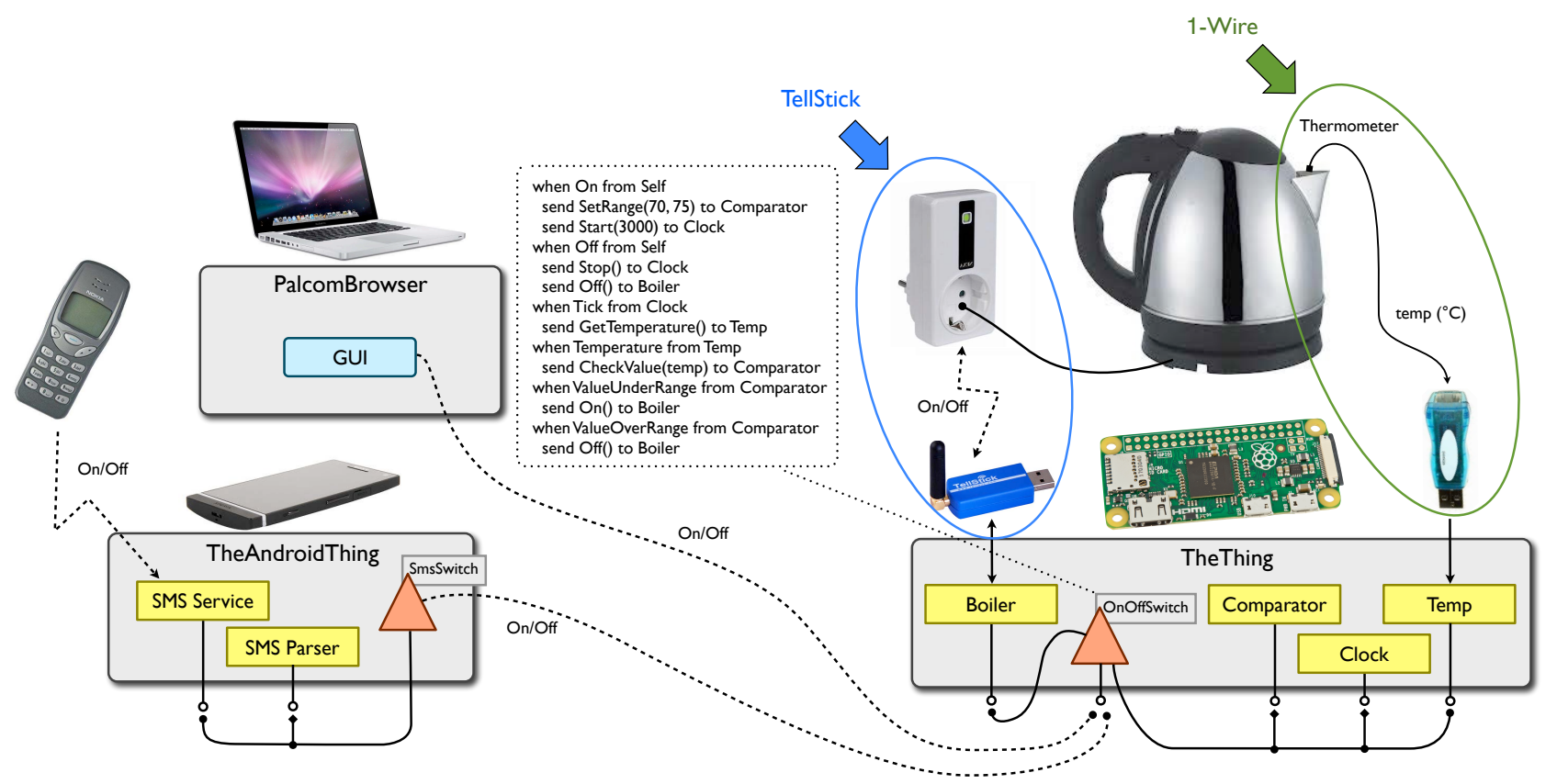

Figure 1: PalCom implementation of remote control of electrical kettle. The glue-code to coordinate the services (yellow boxes) is embedded in the assemblies (orange triangles). Here illustrated in a script-like format.

provide and render a graphical user interface for the commands they provide. After understanding how they work we put together the OnOffSwitch assembly (orange triangle) with the help of the graphical tool provided by the PalcomBrowser. The content of this assembly is outlined in Figure 1 in a script-like format. It shows that it merely consists of event-clauses triggers by incoming messages which will send one or more messages to the services.

During development it is convenient to run it on the PalcomBrowser and when working properly it can be deployed to the TheThing together with the services. The addressing mechanisms of PalCom makes this transfer possible without changing the bindings of the OnOfSwitch assembly.

The OnOffSwitch assembly provides a Service to turn the whole configuration on or off which can be controlled from the PalcomBrowser. Since the intended use case is to activate the kettle remotely we build a second configuration that is deployed on a smartphone which also can control the OnOffSwitch. By using a SMS interface the kettle can be turned on or off from a remote phone by simply sending the agreed on SMS string. As an alternative we can use the Graphical PML Editor [2] to build an app to execute on a remote smartphone that could be used to control the kettle. In that situation it would be tempting to also display the current temperature of the kettle. This would only require a revision of the OnOffSwitch assembly to send temperature reading as commands through its service.

\section{DISCUSSION}

In the kettle example above we built a working system by combining software components from two different vendors, with locally provided services. The logic to combine and coordinate them, the glue, is placed separately, in two assemblies, without any modification to the services. We also note that it did not affect the coordination that some of the software components come from vendors using different communication technologies.

The information to bind assemblies to services on particular devices is embedded in the assemblies which free us from using parameters or other means to configure the services to reference each other. As a result the services are independent of each other and can take part in several configurations or scenarios at the same time. We illustrated that assemblies can be moved between devices, and without change continue to connect to, and communicate with, the services they have been bound to use.

\section{CURRENT AND FUTURE WORK}

Several research projects are working on expanding the expressiveness of the PalCom architecture to meet even more of the challenges we are facing in the IoT era of massively distributed systems. We will here give a short summary of current research topics.

\subsection{Support for Configurations}

In the above examples there are four services and one assembly that we would like to be deployed on the TheThing hosted by the physical device with the attached equipment. We also want to express that the assembly bindings are to services all deployed on the 
same device. The second example, on the smartphone, illustrate that a configuration might have a parameter in order to be useful as a template and used in many instantiations without change. In this case the parameter needed is to the device with the attached equipment. The supplied parameter value is used to bind the dependency to services in the assembly.

\subsection{Support for Systems}

As an example the two configurations in the above example go together, but are deployed on two different devices. In this the case one would need to express that the device where one of the configurations is deployed, is a parameter to the second configuration. We see that the kind of information expressed in Configurations and Systems also is a form of interconnecting code that will be a big advantage to handle separate from the programming itself.

\subsection{Versioning and Updating}

All the components in the PalCom Architecture are versioned. This means that it, at runtime, can support full traceability of all components included in a System. With this as a built in mechanism it supports distributed updating of systems to new versions where only the parts that differ need to be updated. With the PalCom runtime support for executing several versions at the same time we are exploring the possibilities for automatic updating of systems where not all components are available at the same time.

The expressiveness of the coordination in the assemblies is in the first version of this notation very limited, but has still served its purpose surprisingly well. We are, however, now working on a more expressive formalism in order to solve more intricate problems as coordination not having to use the fall-back of introducing a service for the task. We are here also exploring the possibilities for supporting parallelism in the coordination.

Initially PalCom only supported simple parameters to servicecommands. It was, however simple to represent structures as strings using for example JSON and similar formats and thus weakly typed communication. The support for the more efficient PON [5] representation makes it possible to add support for structured types, but still only per command. One option is to introduce the possibility of publishing types for parameters which could then be shared by commands. Naturally such types need to be versioned as well.

Safety is a major concern for all applications. In particular we work with solutions where safety can be achieved both for guaranteeing that the systems we build are not compromised during updating or otherwise, as well as the applications they provide are protected. In an IoT setting which have no apparent human user, part of the challenge is to achieve these goals without complicating the use of the systems too much.

\section{CONCLUSIONS}

We have presented the PalCom architecture and its components and illustrated its use by a small distributed system. We have shown that the architecture supports separation of the parts of system that implement computation, or services, from the parts that implement coordination between these and configuration i.e. the interconnecting code. By achieving this separation we see that the resulting systems become flexible and can be re-arranged without changing the computational parts. Furthermore, the separated coordination mechanisms can be used to retrofit components that were not made to fit, for example come from different vendors, following different standards etc. We see this as a promising way to meet the new software challenges for the emerging programmable world.

\section{ACKNOWLEDGMENTS}

The PalCom architecture was initially formulated in the EU-project Palpable Computing. Since the project PalCom has been completely re-written and extended in many dimensions. We are deeply thankful for all contributions to the system done by many people over the 15 years since it all started.

This work was partially funded by SSF under grant RIT17-0035 SMARTY.

\section{REFERENCES}

[1] Luis Andrade, José Luiz Fiadeiro, Joao Gouveia, and Georgios Koutsoukos. 2002. Separating computation, coordination and configuration. Fournal of software maintenance and evolution: research and practice 14, 5 (2002), 353-369.

[2] Björn A. Johnsson. 2017. Inverted GUI Development for IoT with Applications in E-Health. Ph.D. Dissertation. Department of Computer Science, Lund University. LU-CS-DISS:2017-1. ISBN 978-91-7753-239-2.

[3] Boris Magnusson, Görel Hedin, and Per Runeson. 2018. PalCom MIST: A Metaprotocol for Internet Systems of Things. In 2018 Fifth International Conference on Internet of Things: Systems, Management and Security. IEEE, 206-213.

[4] Peter Middleton, Peter Kjeldsen, and Jim Tully. 2013. Forecast: The internet of things, worldwide, 2013. Gartner Research (2013). https://www.gartner.com/en/ documents/2625419

[5] Mattias Nordahl and Boris Magnusson. 2015. A lightweight data interchange format for Internet of Things in the PalCom middleware framework. Procedia Computer Science 56 (2015), 284-291.

[6] PalCom. 2019. http://www.ist-palcom.org @Online: 2019-06-26.

[7] David Svensson Fors. 2009. Assemblies of Pervasive Services. Ph.D. Dissertation. Department of Computer Science, Lund University. LU-CS-DISS:2009-1. ISBN 978-91-976939-1-2

[8] Antero Taivalsaari and Tommi Mikkonen. 2017. A roadmap to the programmable world: software challenges in the IoT era. IEEE Software 34, 1 (2017), 72-80.

[9] Mark Weiser. 2002. The computer for the 21st century. IEEE pervasive computing 1,1 (2002), 19-25 